\section{Which colours are seen by the patient during cataract surgery? Results of an intraoperative interview}

\begin{abstract}
Purpose To discover what cataract patients see during phacoemulsification and if these light phenomena influence their anxiety levels during surgery.

Methods In all, 200 patients were interviewed intraoperatively at the Eye Hospital, Petrisberg, Trier, Germany. The quality of the visual experiences was described and if these were pleasant, neutral or unpleasant. Systemic sedation was noted.

Results Among 200 patients (209 eyes): 88 were men (91 eyes; 44\%) and 112 were women (118 eyes; 56\%). Median age (years): men (71), women (70). Mean operating time was $8 \mathrm{~min}$. 49/209 (23\%) were not anxious before and during surgery. 110/209 (52\%) were more anxious before than during surgery, 50/209 (24\%) were still anxious during surgery, 27/209 (13\%) got sedation with midazolam (1-5 mg). Colours in descending order seen: blue, red, pink, yellow, green, purple, turquois, and orange. The most dominant colour combination was $\mathrm{red} / \mathrm{blue}$. Structures were seen by $162 / 209$ $(78 \%)$. Most $(61 \%)$ intraoperative visual experiences were pleasant, $38 \%$ were neutral, and $1 \%$ found them transiently unpleasant. Three patients felt blinded by the light of the operating microscope. Conclusions The experience of colours and other light phenomena was pleasant for most patients during phacoemulsification under topical anaesthesia. They occur spontaneously when the patient is fixating on the operating light. They are not dependent on the individual or environment. Sedation only in $13 \%$. Direct questioning for visual sensations by the operating surgeon may lead to less need for sedation and lead to less side effects
\end{abstract}

M Wenzel ${ }^{1,2,4}$ and M Schulze Schwering ${ }^{1,3,4}$

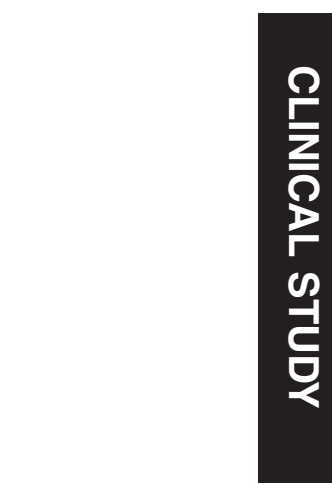

for elderly and multimorbidity people postoperatively. The surgeon can use this knowledge to reassure patients during surgery.

Eye (2016) 30, 385-391; doi:10.1038/eye.2015.239; published online 13 November 2015

\section{Introduction}

In Germany $59 \%$ of all routine cataract operations are performed under intravenous sedation, $15 \%$ with oral sedation, and $29 \%$ using topical anaesthesia without any sedation. ${ }^{1}$ Sedation causes side effects during and after cataract surgery. Known side effects of midazolam as with diazepam are: respiratory depression, coughing, dyspnoea, and less commonly phlebitis. Many cataract patients have visual illusions during their operation that can alarm them.

During the last twenty years there have been several studies of intraoperative visual illusions. ${ }^{2-25}$ These visual phenomena are mostly independent of comorbidities, sex, age, form of anaesthesia, duration of the operation, mode of cataract surgery, or even sedation. Intraoperative visual illusions can lead to anxiety ${ }^{2,10,20}$ or joy. ${ }^{11,24}$ This is why patients are counselled about them prior to surgery. 7,21

This is the first study that examines an intraoperative interview of patients instead of questioning patients postoperatively about light phenomena. Cataract patients were asked about their visual sensations during their surgery under topical anaesthesia. They were asked about any anxiety or pleasure they felt, as well as the colours and structures seen and the clarity of their visual experiences.
${ }^{1}$ Eye Hospital, Petrisberg, Trier, Germany

${ }^{2}$ University Eye Hospital, Aachen, Germany

${ }^{3}$ Department für Augenheilkunde, University of Tübingen, Tübingen, Germany

Correspondence: M Schulze Schwering, Department für Augenheilkunde, University of Tübingen, Schleichstraße 12-16, 72076 Tübingen, Germany Tel: +49 15162450 976; Fax: +497071940213. E-mail: mssoculus@web.de

${ }^{4}$ These authors contributed equally to this work.

Received: 20 April 2015 Accepted in revised form: 30 September 2015 Published online: 13 November 2015 


\section{Materials and methods}

We report the findings from 201 consecutive patients (210 eyes) who underwent ambulant cataract surgery. On the day of the operation the procedure was explained to them taking about $20 \mathrm{~min}$. During this time they were shown a board with 50 , mostly coloured, pictures drawn postoperatively by patients previously operated on (Figures 1 and 2). ${ }^{22}$ We asked them to concentrate on what they might see during the operation. One of the 201 patients was deaf and suffering from dementia and his results were excluded from the study analyses. Our patients did not get routine sedation, but only on request either shortly before or during phacoemulsification.

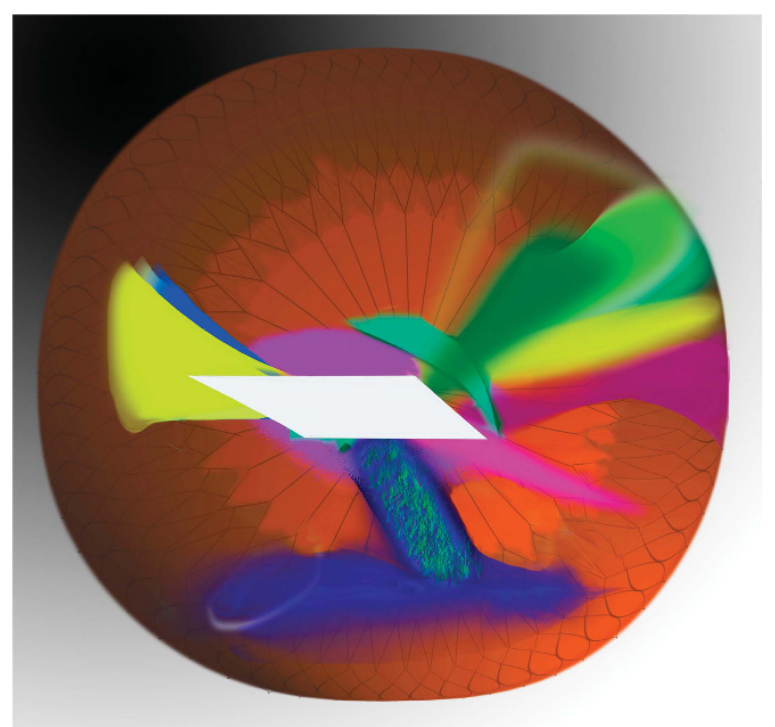

Figure 1 Postoperative drawing about intraoperative visual illusions. More than four colours, intensive, polygonal.

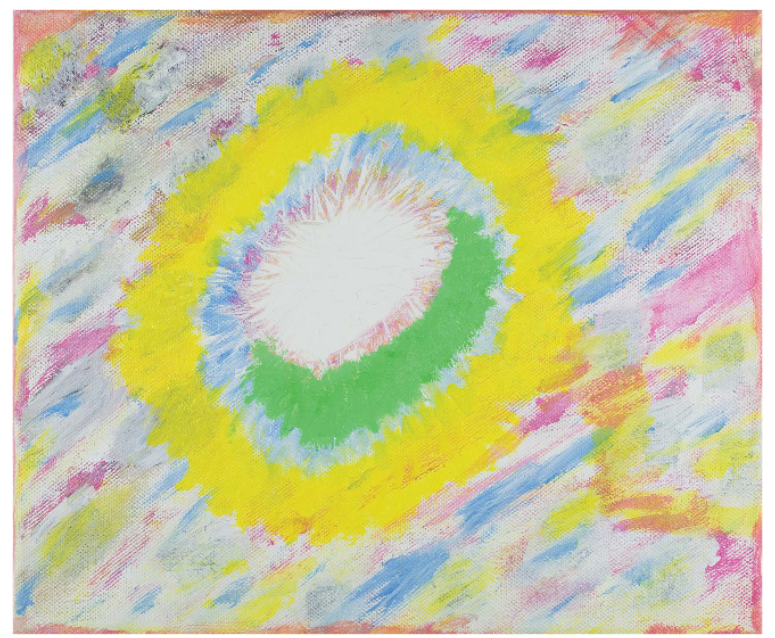

Figure 2 Postoperative drawing about intraoperative visual illusions. More than four colours, pale, polygonal.
Routinely we administered proxymethacain- $\mathrm{HCl}$ 0.5\% eye drops preoperatively for topical anaesthesia and a postoperative subconjunctival injection $0.1 \mathrm{ml}$ xylocaine and adrenaline. The operation was performed using a microscope with adjustable slit beam, mostly of 1-2 mm width that also allows retroillumination (operating microscope by Möller Wedel). Just before beginning the surgery patients see this light from the microscope reflected by a hexagonal mirror. Operating time was 5 - $35 \mathrm{~min}-$ median time $8 \mathrm{~min}$. In five patients operating time was more than $15 \mathrm{~min}$. All procedures were performed by the same surgeon (MW).

Informed consent was obtained from all participants. The study adhered to the tenets of the declaration of Helsinki. At the beginning of phacoemulsification patients were asked by the surgeon if they saw colours or if everything was just white. In addition, if they saw a central light and if it was round, elongate, crystalloid, or a different shape. Patients reported either spontaneously about their visual experiences or they were asked again by the surgeon during the operation or lens implantation. All patients had a capsular fixated hydrophobic acrylic lens (ALCON; Acrysof, USA), imbedded that were $85 \%$ uncoloured and $15 \%$ with blue filter. After the operation patients were asked if they were more anxious before, during, or after surgery. Subsequently the surgeon filled in a standardized questionnaire form on their behalf.

In nine patients surgery of the second eye was performed within 9 month. We are presenting all their 18 surgeries as single procedures.

\section{Results}

In all, 200 patients (209 eyes) took part in our study, 88 men, 91 eyes (44\%) and 112 women, 118 eyes (56\%). Median age in men was 71 and in women 70 years. A total of 111 'first eyes' with a median VA of $0.4(20 / 50)$ were operated and 98 'second eyes' with a median VA of $0.4(20 / 50)$ with implantation of a capsular fixated hydrophobic acrylic lens. Four patients with advanced glaucoma underwent a combined procedure of phacoemulsification and trabeculotomy. In another patient an AC-IOL has been implanted after traumatic cataract. Of 209 advanced cataracts 14/209 (7\%) had pseudoexfoliation syndrome. Other comorbidities in descending order were elevated IOP or POAG 61/209 (29\%), dry ARMD 27/209 (13\%), PACG 12/209 (6\%), macula pucker $11 / 209(5 \%)$, Cornea guttata 6/209 (3\%), and wet ARMD 4/209 (2\%).

\section{Colours}

Eighty-seven percent (184/209) saw colours and 25/209 (12\%) did not; 19 saw only white/bright, 5 white and 
black (dark), and 1 saw white and grey. Blue (74\%), red $(42 \%)$, and pink $(30 \%)$ were the colours seen most often and usually as single colours (Figures $1-3$ ). Colours yellow, green, purple turquois, and orange were hardly seen except with other colours. 39/209 saw one colour, $65 / 209$ two colours, 37/209 three colours, and 43/209 saw four or more colours (eg, like in Figures 1 and 2). Of $64 / 209(31 \%)$ patients who saw no or only one colour 39 (61\%) were men $39 / 88$. Of $145 / 209$ (69\%) patients who saw two or more colours 93 (65\%) were women. Women saw red and pink slightly more often than men (Table 1).

Four of 32 patients saw a change in colour to yellow when a single piece foldable yellow lens had been implanted (ALCON; Acrysof). Operating time was 5-35 min-median $8 \mathrm{~min}$. In 6/209 procedures time was more than $15 \mathrm{~min}$ : one miotic patient, one with posttraumatic cataract and AC IOL implantation, and four patients with combined trabeculotomy. Four of six saw colours: three blue, and of the rest each one saw yellow, orange, or pink. Patients below the age of 60 years saw

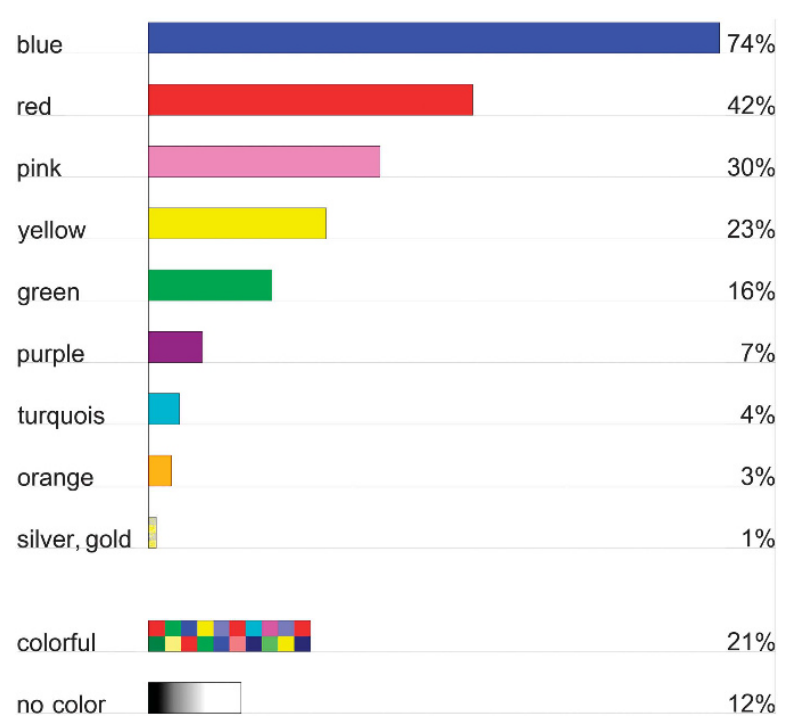

Figure 3 Colours seen by patients intraoperatively. red more than other colours (Table 1). 155/209 (82\%) saw pale colours (like water colours, eg, like Figure 2). 29/209 (14\%) saw intensive colours (like acrylic colours, eg, like Figure 1). 15/209 saw pale and intensive colours at the same time. In $25 / 29$ procedures the intensive colours were pleasant, $4 / 29$ were neutral, and $4 / 29$ were sedated. $18 / 29$ were preoperatively emotionally stressed and 4/29 remained stressed intraoperatively.

Twenty-nine patients had preoperative visual acuity of 0.2 or less. $14 / 29(48 \%)$ saw three or more colours during surgery, most frequently blue, followed by red and pink. Seventy-three patients had glaucoma or an elevated IOP. $27 / 73(36 \%)$ saw three or more colours of which blue was most dominant, followed by red and yellow. There were no differences between elevated IOP and glaucoma patients. Thirty-one patients were suffering from ARMD. 9/31 (29\%) saw three or more colours, mostly blue followed by pink and yellow. Our data does not allow describing significant differences among patients and their specific diseases (Figure 3).

Ninety-eight patients underwent phacoemulsification on their second eye. 61/98 (62\%) remembered the visual illusions of their first operation: 36/61 (59\%) remembered them differently, for $25 / 61$ (41\%) they were similar. 9/98 patients underwent cataract surgery of their second eye, six women and three men. Two women saw four or more colours during both operations. One of them described them more impressive during her first procedure. The other seven patients saw different colours and structures in both surgeries.

\section{Structures}

In 157/209 cases patients recognized at least one of four predetermined structures (circle, oval, hexagonal or polygonal, stripes): A circle by 45 , oval (eg, like Figure 2) by 48 , hexagonal or polygonal by 73 (eg, like Figure 1 ) and stripes by 11 . In 51/209 procedures they saw other structures: 20 described them as like the sky (8), or clouds (7), or sunrise (2) and 3 as one each of the

Table 1 Colours seen by patients intraoperatively plus other psychosocial data of patients

\begin{tabular}{lccccccccc}
\hline In $\%$ & All & Men & Women & Sedation & Intensive & Quality + & Quality 0/- & Anxious & $<60$ years \\
\hline No colours & 12 & 14 & 10 & 26 & - & 0 & 28 & 18 & 13 \\
Blue & 74 & 72 & 75 & 64 & 73 & 80 & 58 & 77 & 32 \\
Red & 42 & 34 & 50 & 45 & 55 & 40 & 42 & 61 \\
Pink & 30 & 23 & 36 & 45 & 9 & 27 & 35 & 12 & 16 \\
Yellow & 23 & 20 & 25 & 27 & 10 & 19 & 11 & 9 \\
Green & 16 & 15 & 18 & 0 & 10 & 42 & 8 & 24 & 16 \\
$\geq 4$ colours & 23 & 21 & 25 & 45 & 62 & 42 & 16 \\
\hline
\end{tabular}

Line 1: 'no colours': patients, who did not see any colour. The \% values are related to all the 209 patients line 2-6: 'blue, red, pink, yelllow, and green': patients who recognized one of these colours. The \% values are related to 141 patients, who recognized one, two, or three colours line $7:$ ' $>4$ colours': patients who recognized four or more colours. The \% values are related to all the 209 patients. 
following: gates of heaven, fireworks, and rays. Twentyone mentioned objects looking like crystals: diamond, crystals, sapphires, jewels, or ice crystals. Further shapes were rings, pebble stones, kaleidoscopes, leaves, and tinsel. Five patients saw details of the operating theatre: the theatre nurse (two) and one each of: the spotlight, a surgical instrument, or a surgical container. The combination or frequency of colours seen did not depend on whether the patient saw a shape or not.

\section{Quality}

In $128 / 209$ (61\%) cases patients found their visual illusions pleasant, 79/209 (38\%) found them neutral and $2 / 209$ (1\%) thought them unpleasant. In 2/209 their feelings changed from unpleasant to pleasant, in another 2/209 from neutral to pleasant. Four did not report on this quality.

Three patients (four procedures $(2 \%)$ ) reported visual illusions as unpleasant. These three were anxious both before and during the operation. Two of the three required sedation with $2 \mathrm{mg}$ of dormicum and saw only bright light intraoperatively, with no colours or shapes. The third patient with two procedures did not want sedation at all. In both operations he saw blue with pink in the first and with red in the second procedure. During the first surgery he was blinded by the light of the operating microscope, which disappeared when the slit beam size was reduced to $1 \mathrm{~mm}$. During the second surgery he first saw red, which he described as unpleasant but this changed to a pleasant when blue. Another eight patients received cataract surgeries in both eyes during the study period. In four visual illusions were pleasant in both eyes. The other four recognized them mostly as pleasant or neutral.

\section{Excitement and sedation}

During forty-nine procedures (23\%) patients were not anxious preoperatively. 110/209 (53\%) were tense before the operation and became more relaxed during the procedure. 50/209 (24\%) were equally tense both before and during the operation. In none of 209 procedures patients were more nervous during than before the operation. Tense patients saw slightly more red than pink (Table 1). 27/209 asked for sedation (13\%): 4/209 received $0.5 \mathrm{mg}$ midazolam, 11/209 received $1.0 \mathrm{mg}$ and 2/209 got $1.5 \mathrm{mg}$. Eight people received $2 \mathrm{mg}$ midazolam and three received $5 \mathrm{mg}$. The mean age of the 29 patients who received sedation was 70 years; 8 men and 19 women. One wanted sedation although he was not anxious. Seventeen wanted sedation and became much more calm during the procedure. Nine got sedation and remained anxious during the operation even when the, dose of sedation was increased. In 92/209 procedures patients were anxious before the operation and became calm during the procedure without sedation. All 15 patients with a low dose of sedation (0.5-1 mg midazolam) saw colours, in contrast to 5/12 with higher dosages of sedation ( $>1.5 \mathrm{mg}$ midazolam). Of 25 patients who did not see any colour, $7 / 25$ (28\%) got $1.5-2 \mathrm{ml}$ midazolam for sedation. Sedated patients mostly saw no colour or lots of colours. They tended to see less blue and green and more pink and yellow (Table 1). Seven of 27 sedated patients saw no colours, 2 saw one, 6 two, 3 three, and 9 saw four or more colours (Table 1 ).

\section{Discussion}

For the first time cataract patients have been asked about their visual experiences during surgery using topical anaesthesia. Asking about their experience during surgery reassured even anxious patients and only $13 \%$ needed sedation with midazolam $1-5 \mathrm{mg}$ intravenously. Talking to patients about light phenomena reduced their anxiety and distracted them from the actual situation. For $61 \%$ of our patients the quality of visual experiences was pleasant, for $38 \%$ neutral and for $1 \%$ unpleasant. Thus it seems not only to reduce the need for general anaesthesia but is also a safe method to do phacoemulsification.

It was interesting to observe that patients saw proportionatlely similar colours according to those we described in 'Visual experiences under topical anaesthesia' in which we focused on the patients' recognition of pictures, which had been previously painted postoperatively by other patients to describe their visual impressions (Figures 1 and 2). ${ }^{22}$

Intravenous midazolam did not seem to significantly reduce pain or anxiety. It must be remembered that benzodiazepines themselves may induce anxiolysis, amnesia, and sedation. They are both sedative and anticonvulsive. They work by binding to GABA receptors. Known side effects of midazolam and diazepam are respiratory depression, coughing, and dyspnoea, and more rarely phlebitis. Rarely a paradoxical reaction can be observed characterized by aggressiveness, hostility, and restlessness. With oral benzodiazepines this occurs in about $5 \%$ of patients.

The more colours patients see during the operation the more pleasant the operation is for them. For none of the patients the colours that they saw intraoperatively remained unpleasant. Three were temporarily blinded, one found red unpleasant for him, but seeing blue during the operation was reported as pleasant.

Investigators from UK and Asia report that the operating light was disturbing at the beginning of the operation. $4,5,14$ In our study only $1 \%$ of patients complained about it. This might be influenced by the fact 
Table 2 Comparison of intraoperatively colours seen in different studies

\begin{tabular}{|c|c|c|c|c|c|c|c|c|c|}
\hline Author & Murdoch & AuEong & AuEong & AuEong & Newman & Prasad & Rengaraj & Wenzel & Wenzel \\
\hline Year & 1994 & 1999 & 2000 & 2000 & 2000 & 2003 & 2004 & 2014 & 2015 \\
\hline $\mathrm{N}$ & 56 & 100 & 70 & 52 & 106 & 121 & 306 & 100 & 209 \\
\hline Country & UK & Singapore & Singapore & Singapore & UK & UK & India & Germany & Germany \\
\hline Anaesthesia & Peribulbar & Retrobulbar & Retrobulbar & Topical & Topical & Subtenon & Topical/retrobulbar & Topical & Topical \\
\hline OP & ECCE & ECCE & Phaco & Phaco & Phaco & Phaco & Phaco & Phaco & Phaco \\
\hline Min & - & 24 & 40 & 20 & - & - & 7 & 8 & 8 \\
\hline Sedation & None & Oral & Oral & Oral & None & None & None & (None) & (None) \\
\hline Age & 72 & 68 & 65 & 68 & 79 & - & 57 & 71 & 71 \\
\hline Colours (\%) & 80 & 56 & 56 & 98 & 69 & 45 & $61-83$ & 74 & 88 \\
\hline Blue & 24 & 20 & 44 & 24 & 37 & 20 & $46-51$ & 49 & 57 \\
\hline Red/pink & 29 & 52 & 59 & 48 & 21 & 11 & $19-20$ & 43 & 54 \\
\hline Yellow & 16 & 52 & 31 & 46 & 8 & 5 & - & 36 & 17 \\
\hline Green & - & 21 & 18 & 14 & 10 & - & $5-13$ & 16 & 13 \\
\hline Orange & - & 4 & 3 & 12 & 12 & - & 7 & 8 & 2 \\
\hline Multiple & - & 27 & 21 & 16 & 10 & - & $<1$ & 16 & 23 \\
\hline
\end{tabular}

that we are operating with a confocal slit beam of 1-2 mm that also allows retroillumination (operating microscope by Möller Wedel).

Questioning about colours intraoperatively makes one realize that colours are more intense than when described after the operation. In all, $88 \%$ of our patients reported colours when interviewed during the operation but only $74 \%$ when interviewed afterwards. ${ }^{22}$ This may be influenced by the having been questioned about colours only to realize for the first time that there were colours and then concentrate on them during the ongoing of the cataract surgery. This may make them calmer. We observed this positive effect also on patients who were moving their eyes a lot: once they have been told to concentrate on the colours that they saw, the eye became more stable. When asking if colours had been pleasant during the operation, $60 \%$ of the ones who were interviewed intraoperatively reported them as pleasant but $50 \%$ when asked later.

Women noticed more colours (median $=3$ ) than men (median $=2$ ). In all groups blue was the dominant colour, followed by red and pink. Blue tended to be found more pleasant than red or pink. Sedation often led to no colours being seen or to $\geq 4$ colours. Our data suggests that patients who do not see any colours need more sedation. With sedation patients see a lot of colours that make the operation more pleasant to them. A study with only sedated patients is difficult because retrograde amnesia might affect recall. All shapes seen, such as a circle, oval, or hexagon can be explained by the position and shape of the operating light.

The majority of patients, who were having a second eye operated, had different visual experiences than during their first operation. We conclude that the visual images may be independent of other factors of the patient.
During the last 20 years several studies of intraoperative visual illusions have been carried out (Table 2). ${ }^{2-25}$ There are great differences in the outcome of these studies and no understanding of the importance or significance of these different visual phenomena. In contrast to former studies we took a new approach of an intraoperative instead of a postoperative interview and discussed these visual effects with the patient reassure and make the operation more interesting to him.

It has been known that patients who undergo a cataract operation under retro or parabulbar anaesthesia see less colours than those who are operated using topical anaesthesia. It is striking that the colour mostly seen in India and Europe is blue, whereas in Singapore and China it was red and yellow. Longer operating times reported from Singapore do not seem to be responsible. In our own study none of the six patients with an operating time longer than 15 min saw red intraoperatively. We also do not think that routine oral sedation was the underlying reason. Sedated patients in our own study saw red and yellow slightly more often than non-sedated patients. Blue is the colour most commonly seen regardless of which anaesthesia the patient underwent.

Studies concerning visual sensations during intraocular surgery are difficult as such sensations may change rapidly within the same. When being asked about their visual sensations patients report rather colours than structures. Few patients reported them to be similar to those after taking lysergic acid diethylamide, a psychedelic drug, which might be a hint that they are generated by the visual cortex rather than from the retina.

We conclude that the experience of colours and other light phenomena was a pleasant side effect for most patients during phacoemulsification using topical anaesthesia. They occur spontaneously when the patient 
is fixating on the operating light. They are independent on other cofactors. In our study only $13 \%$ needed sedation. Active questioning for visual sensations by the operating surgeon during the operation may lead to a reduction in the need for sedation with less drug side effects postoperativley for the elderly and those with multimorbidity.

\section{Summary}

What was known before

- Intraoperative visual illusions can lead to anxiety or joy. This is why patients are counselled about them before surgery.

- Patients who undergo a cataract operation under retro or parabulbar anaesthesia see less colours than those who are operated using topical anaesthesia.

\section{What this study adds}

- Experience of colours and other light phenomena was a pleasant side effect for most patients during phacoemulsification using topical anaesthesia. They occur spontaneously when the patient is fixating on the operating light. They are independent on other cofactors. In our study only $13 \%$ needed sedation.

- Active questioning for visual sensations by the surgeon himself during the operation may lead to a reduction in the need for sedation with less drug side effects postoperativley for the elderly and those with multimorbidity.

\section{Conflict of interest}

The authors declare no conflict of interest.

\section{Author contributions}

MW collected the data. MW and MSS designed the study, did the study analysis, edited the manuscript for submission, reviewed the manuscript before submission, and agreed to manuscript being published.

\section{Acknowledgements}

We thank Professor Elizabeth Molineux from Great Britain, Head of Paediatric Oncology, College of Medicine, Blantyre, University of Malawi, for crossreading the first English version of the paper. We also thank Mrs Lüer-Kirsch, University of Mainz, for allowing us to publish her intraoperative painting (Figure 1).

\section{References}

1 Wenzel M, Kohnen T, Scharrer A, Schayan K, Klasen J. Ambulante Intraokularchirurgie Ergebnisse der Umfrage 2009 von BDOC, BVA und DGII. Ophthalmo-Chirurgie 2010; 22: 276-283.
2 Ang CL, Au Eong KG, Lee SS, Chan SP, Tan CS. Patients' expectation and experience of visual sensations during phacoemulsification under topical anaesthesia. Eye (Lond) 2007; 21(9): 1162-1167.

3 Au Eong KG, Lee HM, Lim AT, Voon LW, Yong VS. Subjective visual experience during extracapsular cataract extraction and intraocular lens implantation under retrobulbar anaesthesia. Eye (Lond) 1999; 13(Pt 3a): 325-328.

4 Au Eong KG, Lim TH, Lee HM, Yong VS. Subjective visual experience during phacoemulsification and intraocular lens implantation using retrobulbar anaesthesia. J Cataract Refract Surg 2000; 26: 842-846.

5 Au Eong KG, Low CH, Heng WJ, Aung T, Lim TH, Ho SH et al. Subjective visual experience during phacoemulsification and intraocular lens implantation under topical anaesthesia. Ophthalmology 2000; 107(2): 248-250.

6 Chung CF, Lai JS, Lam DS. Visual sensation during phacoemulsification and intraocular lens implantation using topical and regional anaesthesia. J Cataract Refract Surg 2004; 30(2): 444-448.

7 Haripriya A, Tan CS, Venkatesh R, Aravind S, Dev A, Au Eong KG. Effect of preoperative counseling on fear from visual sensations during phacoemulsification under topical anaesthesia. J Cataract Refract Surg 2011; 37(5): 814-818.

8 Khan A, Conley A. An explanation for patient intraocular instrument visualization. J Cataract Refract Surg 2005; 31(1): 237-238.

9 Kluxen G. Entoptische Phänomene, optische Täuschungen und andere außergewöhnliche Seheindrücke. In: Ungeahnte Bilder. Kluxen G. (Hrsg). Kaden Verlag: Heidelberg, Germany, 2004.

10 Laude A, Au Eong KG, Mills KB. Knowledge of visual experience during cataract surgery under local anaesthesia: a nationwide survey of UK ophthalmologists. $\mathrm{Br} J$ Ophthalmol 2009; 93(4): 510-512.

11 Malik A, Kumaran N, Zia R. An insight into patient visual experiences during cataract surgery. Br J Ophthalmol 2010; 94(10): 1401-1402.

12 Malik A, Kumaran N, Zia R. An insight into patient visual experiences during cataract surgery. Authors' response. Br J Ophthalmol 2011; 95(6): 895.

13 Murdoch IE, Sze P. Visual experience during cataract surgery. Eye 1994; 8: 666-667.

14 Newman DK. Visual experience during phacoemulsification cataract surgery under topical anaesthesia. $\mathrm{Br} J$ Ophthalmol 2000; 84(1): 13-15.

15 Prasad N, Kumar CM, Patil BB, Dowd TC. Subjective visual experience during phacoemulsification cataract surgery under sub-Tenon's block. Eye 2003; 17: 407-409.

16 Rengaraj V, Radhakrishnan M, Au Eong KG, Saw SM, Srinivasan A, Mathew J et al. Visual experience during phacoemulsification under topical versus retrobulbar anaesthesia: results of a prospective, randomized, controlled trial. Am J Ophthalmol 2004; 138(5): 782-787.

17 Sumich PM, Francis IC, Kappagoda MB, Alexander SL. Artist's impression of endocapsular phacoemulsification surgery. J Cataract Refract Surg 1998; 24: 1525-1528.

18 Tan CS. Patients experience different types of visual sensations during cataract surgery. Br J Ophthalmol 2011; 95(12): 1758-1759.

19 Tan CS, Au Eong KG, Kumar CM. Visual experiences during cataract surgery: what anaesthesia providers should know. Eur J Anaesthesiol 2005; 22(6): 413-419.

20 Tranos PG, Wickremasinghe SS, Sinclair N, Foster PJ, Asaria R, Harris ML et al. Visual perception during 
phacoemulsification cataract surgery under topical and regional anaesthesia. Acta Ophthalmol Scand 2003; 81(2): 118-122.

21 Voon LW, Au Eong KG, Saw SM, Verma D, Laude A. Effect of preoperative counseling on patient fear from the visual experience during phacoemulsification under topical anaesthesia: Multicenter randomized clinical trial. J Cataract Refract Surg 2005; 31(10): 1966-1969.

22 Wenzel M, Sigmann D, Schulze Schwering M. Visual experiences during cataract surgery under topical anaesthesia. Ophthalmologe 2014; 111(11): 1065-1069.
23 Wickremasinghe SS, Tranos PG, Sinclair N, Andreou PS, Harris ML, Little BC. Visual perception during phacoemulsification cataract surgery under subtenons anaesthesia. Eye 2003; 17: 501-505.

24 Zia R, Schlichtenbrede FC, Greaves B, Saeed MU. "Only rarely seen in dreams"-visual experiences during cataract surgery. Br J Ophthalmol 2005; 89(2): 247-248.

25 Sugisaka E, Shinoda K, Sano RY, Ishida S, Imamura Y, Ozawa $\mathrm{Y}$ et al. Mechanism of visual sensations experienced during pars plana vitrectomy under retrobulbar anaesthesia. Ophthalmologica 2010; 224(2): 103-108. 